\title{
A Comparative Study of Three Imaging Modalities for the Size Selection of Left Atrial Appendage Closure Device
}

\section{Zhong-bao Ruan}

Jiangsu Taizhou People's Hospital https://orcid.org/0000-0003-0607-0010

\section{Fei Wang}

Jiangsu Taizhou People's Hospital

Gecai Chen

Jiangsu Taizhou People's Hospital

Jun-guo Zhu

Jiangsu Taizhou People's Hospital

Yin Ren

Jiangsu Taizhou People's Hospital

Wei Li

Jiangsu Taizhou People's Hospital

Kai Jin

Jiangsu Taizhou People's Hospital

Li Zhu ( $\nabla$ tzheart@163.com)

Jiangsu Taizhou People's Hospital

\section{Research Article}

Keywords: Left atrial appendage closure, Non-valvular atrial fibrillation, CT angiography, transesophageal echocardiography, digital subtraction angiography.

Posted Date: August 19th, 2021

DOl: https://doi.org/10.21203/rs.3.rs-814153/v1

License: (c) (i) This work is licensed under a Creative Commons Attribution 4.0 International License.

Read Full License 


\section{Abstract}

Background Accurate preprocedural measurements is important for left atrial appendage closure (LAAC). However, there were limited data comparing tomography angiography (CTA), trans-esophageal

echocardiography (TEE) and digital subtraction angiography (DSA) for diagnostic accuracy and the utility for LAAC. This study aimed to compare the results of CTA, TEE and DSA measurements and analyze their accuracy, correlation and consistency in patients who successfully underwent LAAC.

Methods 157 non-valvular atrial fibrillation (AF) patients who underwent LAAC were included. The maximum diameter and depth of LAA were recorded by CTA, TEE and DSA. Correlations and agreements were compared.

Results All patients were successfully performed LAAC with Watchman device. There was no significant difference in the diameter measurement of LAA ostium between DSA and TEE. Meanwhile, The diameter of LAA ostium obtained by CTA was greater than that of DSA and TEE. There were good correlations between the LAA ostium measured by TEE, CTA, DSA and the Watchman device. DSA measurements and the actual device size were the widest limits of agreement, followed by TEE, CTA measurements were the narrowest limits of agreement. For the LAA depth measurements, mean CTA measurements was higher than that of TEE and DSA. There was no significant difference in the depth measurement among the three imaging modalities.

Conclusions CTA, TEE and DSA measurements had good correlations with WATCHMAN device. The ostium diameter and depth of the LAA measured by CTA were greater than those measured by TEE and DSA. The relevance and concordance of CTA measurements were the best.

\section{Background}

Non-valvular atrial fibrillation (AF) is the most common clinical arrhythmia and AF-associated stroke is the most fatal complication [1]. The main source of thrombus in patients with AF at high stroke risk is left atrial appendage (LAA) [2]. Due to the contraindications and disadvantages of anticoagulants, left atrial appendage closure (LAAC) has been shown to be an effective method to prevent stroke in AF patients with high stroke risk [3]. It is a key procedure to fully evaluate the shape and depth of the LAA. Meanwhile, it is essential to obstain a optimal LAA imaging and sizing to improve procedural and successful LAAC. Underersizing of the occluder may result in large amounts of residual leakage around the device and evendevice-related thrombus (DRT) formation, whereas oversizing of the occluder carries the risk of cardiac tampondsa [4]. CT angiography (CTA), transesophageal echocardiography (TEE) and intraoperative digital subtraction angiography (DSA) were usually used to assess LAA size and shape before LAAC. However, there were limited data comparing CTA, TEE, and DSA for diagnostic accuracy and the utility for LAAC. Therefore, we sought to compare the results of CTA, TEE, and DSA measurements and analyze their accuracy,correlation and consistency in patients who successfully underwent LAAC. 


\section{Methods}

\section{Study population}

A total of 157 consecutive patients with AF who underwent LAAC in Taizhou People's Hospital from May 2019 to June 2020 were included in the study. The diagnosis of AF was mainly based on the criteria listed in the 2016 ESC Guidelines for the management of AF developed in collaboration with EACTS [5]. Indications for LAAC were AF with high stroke risk (CHADS-VASC $\geq 2$ in male or CHADS-VASC $\geq 3$ in female) and contraindications to OAC [6]. The baseline clinical characteristics were collected including age, gender, CHA2DS2-VASc score, HAS-BLED score, comorbidities, medical history, left ventricular ejection fraction (LVEF), perioperative adverse events, LAA measurements, and postoperative follow-up were collected. This study was approved by the Ethics Committee of JiangsuTaizhou People's Hospital. Written informed consent for the procedure and data collection was obtained from all patients before entering this experiment.

\section{Pre-procedural protocols}

TEE and CTA were performed 24 hours before LAAC to exclude the thrombus in the left atrium (LA) or LAA and measure the data of the ostial dimensions and depths of LAA. TEE was performed using Philips EPIQ 7C ultrasound. LAA morphology, lobulation and thrombus were observed from approximate TEE angles of $0^{\circ}, 45^{\circ}, 90^{\circ}$ and $135^{\circ}$ in the LA at the end of systolic period. The maximum orifice diameter and depth of LAA were measured (Figure 1). CTA was performed using 128-slice dual-source CT (SOMATOM Force, Siemens, Germany). LA and LAA were reconstructed at greatest dimension at $250 \mathrm{~ms}$ after the R wave. The maximum LAA ostium diameter and depth were measured by adjusting the orthogonal plane to create a cross-sectional image of the LAA ostium on the oblique coronal images, using the medial side of the left superior pulmonary vein ridge and the left circumflex branch as the segmentation basis (Figure 2).

\section{Procedural protocols}

The patients were fasted for 6 hours before operation. All operations were performed under deep intravenous anesthesia or general anesthesia and TEE monitoring. TEE was performed as described previously. The best position was selected according to the reconstructed CTA images, and the best image was selected in the right anterior oblique (RAO) $0^{\circ} \sim 40^{\circ}$ and caudal (CAU) $0^{\circ} \sim 40^{\circ}$, usually in the angle of $\mathrm{RAO} 30^{\circ}$ andCAU $20^{\circ}$. Then, the maximum diameter and the depth were measured by DSA (Fig. 3). According to the measured diameter, depth, and shape of LAA under CTA, DSA and TEE, an 4-6 $\mathrm{mm}$ upsizing device above the largest diameter was chosen to ensure stable positioning and proper compression. A long sheath of $14 \mathrm{~F}$ was sent into the LAA. Subsequently, the Watchman device was carefully delivered in the LAA through the access system and released at the ostium of the LAA. Before released, the PASS principle was assessed by the device's position; stable anchoring was confirmed by the tug test, an appropriate compression ratio (10\%-25\% of the device original size), and complete 
sealing (residual flow $\leq 3 \mathrm{~mm}$ ) [7]. Once released, TEE and LA angiography were performed to reconfirm device implantation and evaluate complications such as pericardial effusion.

\section{Post procedure}

patients were treated primarily with anticoagulants (warfarin, dabigatran or rivaroxaban) and aspirin or clopidogrel for three months, followed by aspirin and clopidogrel for another three months, then aspirin or clopidogrel for lifelong. Clinical follow-up was performed in the first, third, sixth, and 12th months, then annually. CTA and TEE were reexamined at three months after LAAC. Device positioning, residual leakage, DRT, endothelialization of the occluder and other complications were evaluated.

\section{Statistical Analysis}

Statistical software SPSS 26.0 was used for analysis. Kolmogorov-Smirnov test was used to test whether the data were in normal distribution. The continuous data in normal distribution were expressed as mean \pm standard deviation, and the continuous data in non-normal distribution were expressed as median \pm interquartile range. Three radiographic measurements were compared using a t-test. The correlation of CTA, TEE, and DSA measurements of left atrial appendage ostium diameter and depth was analyzed by Spearman analysis, and agreement was achieved by regression of the deviation and mean of the two measurements of the same sample using a Bland-Altman plot (drawn by MedCalc 19.3.1 software), and the limit of agreement (Limits of agreement) was plotted as an indicator of agreement. $p<0.05$ was used as the basis for determining statistical significance.

\section{Results}

\section{Baseline characteristics}

A total of 157 patients underwent LAAC with Watchman device from March 2018 to November 2020 in Jiangsu Taizhou People's Hospital were enrolled. All patients had contraindications to long-term OAC.The median age was 67 years. Persistent and permanent AF was present in 114 patients (72.6\%), whereas 38 (24.2\%) had a history of stroke/transient ischemic attack(TIA). The mean CHA2DS2-VASc score was 4 (36) and HAS-BLED score was $3(2-5)$. The baseline demographics and clinical parameters were detailed in Table 1.

\section{LAAC procedures and parameter analysis}

The procedural details were shown in Table 2. Deep intravenous anesthesia was used in 101(63.7\%) patients and general anaesthesia in $56(36.3 \%)$ cases. All procedures were performed under TEE guidance. Primary technical success of LAAC was achieved in all cases with the Watchman device. The device was deployed at the first attempt in $142 / 157$ cases (90.4\%). After successful device release, mild leak ( $<3 \mathrm{~mm}$ ) was observed in 14 cases and moderate leak $(3-5 \mathrm{~mm})$ in 4 cases. The perioperative adverse event occurred in 5 cases (3.2\%). including 2 pericardial effusions (1.3\%) and 3 vascular complications (1.9\%). Pericardial effusion requiring pericardiocentesis was happened in 1 case. 


\section{The ostium diameters of LAA measured by CTA, TEE, and DSA}

There was no significant difference in the diameter measurement of the LAA ostium between DSA and TEE $[\otimes 22.9 \pm 3.7 \nabla \mathrm{mm}$ vs. $(22.5 \pm 3.6) \mathrm{mm}, \mathrm{q}=0.92, \mathrm{p}=0.16]$. Meanwhile, The diameter of the LAA ostium obtained by CTA $[(24.6 \pm 3.7) \mathrm{mm}]$ was greater than that of DSA and TEE $q \mathrm{q}=3.92, p<0.01 ; q=4.58$, $p<0.01$, respectively $\rrbracket$.

For the measurements of the LAA ostium , there were reasonably good correlations among the three modalities, with $r=0.78$ and $p<0.001$ between TEE/CTA, $r=0.85$ and $p<0.01$ between TEE/DSA, $r=0.77$ and $p<0.001$ between CTA/DSA (Figure 4:A-C ). There were also good correlations between the LAA ostium measured by TEE, CTA, DSA and the Watchman device, with $r=0.85$ and $p<0.01, r=0.92$ and $p<0.01, r=0.79$ and $p<0.01$, respectively(Figur 4:D-F). Among which, the relevance of CTA measurements was the best.

Bland Altman diagram was used to analyze the consistency. The results showed that the $p$ values of CTA versus DSA, CTA versus TEE, TEE versus DSA were $0.0855,0.0663$ and 0.9916 respectively $(p>0.05)$, that is, the difference and mean value were independent, and the consistency limit could be calculated. Compared with DSA, $6.25 \%$ (5 / 80) of CTA was outside the consistency limit, and the maximum difference / mean value within the limit was $38.68 \%$ (8.8 / 22.75). Compared with TEE, $7.5 \%$ (6 / 80) of CTA were outside the consistency limit, and the maximum difference / mean value within the limit was $35.6 \%$ (8.1 / 22.75). The error of comparison between CTA and TEE was beyond the clinically acceptable range $( \pm 25 \%)$. Compared with DSA, $3.75 \%$ (3 / 80) of TEE were outside the consistency limit, the maximum difference / mean value within the limit was $22.52 \%$ (5 / 22.20), the error was within the clinical allowable range $( \pm 25 \%)$ (Figure 5: A-C ). Combined with correlation analysis, the correlation coefficient of TEE and DSA was $r=0.86$, so it can be considered that the results measured by the two methods have good consistency, and the two methods can be used instead of each other in clinic.

DSA measurements and the actual device size were the widest limits of agreement $(-9.3120 \mathrm{~mm}$, $0.2395 \mathrm{~mm})$, followed by TEE $(-9.4445 \mathrm{~mm},-1.0205 \mathrm{~mm})$, and CTA measurements were the narrowest limits of agreement(-8.7912mm, $-1.8724 \mathrm{~mm})$. The $p$-values of the regression analysis of DSA,TEE and CTA with occluder were $0.0003,0.008$ and 0.3102 , the p-values of TEE and DSA were statistically significant (Figure5:D-F).Therefore, the CTA measurements more closely to the actual Watchman device size.

For the LAA depth measurements, mean CTA measurements $(25.1 \pm 3.9 \mathrm{~mm})$ was higher than that of TEE $(24.6 \pm 3.7 \mathrm{~mm})$ and DSA $(23.9 \pm 3.6 \mathrm{~mm})$. There was no significant difference in the depth measurement between CTA and DSA, CTA and TEE, TEE and DSA $(p>0.05)$. The correlations between TEE and CTA $(r=0,66, p<0.01)$, TEE and DSA $(r=0.75, p<0.01)$, CTA and DSA $(r=0.59, p<0.01)$ were good and statistically significant (Figure6:A-C).

\section{Discussion}


LAAC is a efficacy and safety treatment strategy to prevent stroke in patients with AF and has been recommended by guidelines $[8,9]$. Preoperative understanding of the patient's LAA anatomy is helpful for selecting the appropriate occluder and promoting the safety and effectiveness of LAAC. Undersizing of the device or oversizing of the device may cause to adverse events of device unstability, appendage leakage, sealing, or device embolisation [4].

CTA, a non-invasive modality with the superior spatial resolution, arbitrary scans and three-dimensional assessment, is increasingly perfornmed for baseline imaging and post-LAAC imaging. The full scan of angles was visualized and measured on CTA. TEE is a invasive method and the images were operatordependent. Meanwhile, TEE images for Watchman implantation were measured at four angles of $0^{\circ}, 45^{\circ}$, $90^{\circ}$, and $135^{\circ}$. Thus, even though TEE is a conventional modality recommended for LAA measurements and the selection of device, the maximal dimension and dept measured with TEE were perhaps not the true, which may lead to oversizing or undersizing. DSA is an another valuable reference measurement for LAAC besides CTA and TEE. However, multiple angiogram projections were not routinely performed to obtain the true maximal dimensions and depths of the LAA in all patients. As a result, according to the DSA measurements, the device size selection of $16 \%$ patients were not utilized, which may lead to undersizing and adverse clinical events [10].

At present, CTA, TEE and DSA were recommended to measure the orifice diameter and depth of LAA for selecting the appropriate size of a closure device. However, the diameter and depth of the LAA measured by the above three imaging modalities are not consistent. The relationships among the three imaging modalities are not clear. The relationships between the measured values of each method and the size of occlude are also not clear. Previous results suggested that the LAA ostial size measured by CTA was larger than that of TEE and DSA $[10,11]$. Reports by Chow et al indicated that the narrowest limits of agreement were shown in the correlation of the device size and CTA measurements, the choice of the left atrial appendage occluder size based on the CTA measurements was more accurate than the conventional TEE [12].

Results in this study indicated that the correlation among the three imaging methods (CTA, TEE and DSA) was good. Meanwhile, there were also good correlations between the LAA ostium measured by TEE, CTA, DSA and the Watchman device, which indicated that the three modalities might provide valuable reference measurements for the selection of Watchman closure device.In the present study, we further compared the accuracy of different LAA imaging and sizing modalities and aimed to obtain optimal modalities for the selection of percutaneous LAA closure. Our results showed that the ostium diameter and depth of the LAA measured by CTA were greater than those measured by TEE and DSA. On the other hand, there was no significant difference in the diameter measurement of the LAA ostium between DSA and TEE. CTA measurements were the narrowest limits of agreement with Watchman device. The results were consistent with the reports by Saw et al [9]. According to our results, among the three imaging modalities, the relevance and concordance of CTA measurements were the best.

\section{Limitations}


This study still has the following limitations, first of all, it is a single-center, retrospective study with a small sample size and a potential for patient selection bias $\square$ The reliability of the data and results needs to be further substantiated by a larger sample size study. Secondly, for different forms of left atrial appendage, there may be errors in measurement. A further large sample size is therefore required to validate the conclusions of this study.

\section{Conclusion}

LAA morphology is variable and it is important to rationally apply radiographic accurate measurements to select the appropriate closure device model. The size of LAA measured by CTA, TEE and DSA has good correlation with the model of implanted occluder, which can effectively guide the choice of the model of occluder. The ostium diameter and depth of the LAA measured by CTA were greater than those measured by TEE and DSA. Among the three imaging modalities, the relevance and concordance of CTA measurements were the best.

\section{Declarations}

\section{Acknowledgements}

The study was supported by Jiangsu Provincial Medical Innovation Team (Grant No. CXTDB2017015), Jiangsu Commission of Health, China (Grant No. H201665) and the Six Talent Foundation of Jiangsu Province, China (Grant No. WSN-20) .

\section{Compliance with ethical standards}

Written informed consent was obtained from all patients

\section{Conflicts of Interest}

The authors indicated no potential conflicts of interest.

\section{Ethics approval and consent to participate}

This study was approved by the Ethics Committee of Jiangsu Taizhou People's Hospital, China (Approval No. TZHEC2019-13).

\section{References}

1. Kapur S, Mansour M (2014) Left Atrial Appendage Closure Devices For Stroke Prevention. Arrhythm Electrophysiol Rev 3: 25-29.

2. Harada M, Koshikawa M, Motoike Y, IchikawaT, SugimotoK, Watanabe E Ozaki Y (2018) Left Atrial Appendage Thrombus Prior to Atrial Fibrillation Ablation in the Era of Direct Oral Anticoagulants. Circ 
J 82: 2715-2721.

3. Reddy VY, Doshi SK, Kar S, Gibson DN, Price MJ, Huber K, Horton RP, Buchbinder M, Neuzil P, Gordon NT, Holmes DR Jr;. PREVAIL and PROTECT AF Investigators (2017) 5-Year Outcomes After Left Atrial Appendage Closure: From the PREVAIL and PROTECT AF Trials. J Am Coll Cardiol 70: 2964-75.

4. Schellinger PD, Tsivgoulis G, Steiner T, , Köhrmann M (2018) Percutaneous Left Atrial Appendage Occlusion for the Prevention of Stroke in Patients with Atrial Fibrillation: Review and Critical Appraisal. J Stroke. 2018; 20: 281-291.

5. Kirchhof P, Benussi S, Kotecha D, Ahlsson A, Atar D, Casadei B, Castella M, Diener HC, Heidbuchel H, Hendriks J, Hindricks G, Manolis AS, Oldgren J, Popescu BA, Schotten U, Van Putte B, Vardas P; ESC Scientific Document Group (2016) ESC Scientific Document Group.. 2016 ESC Guidelines for the management of atrial fibrillation developed in collaboration with EACTS. Eur Heart J. 2016; 37:28932962.

6. Segers VFM, Heidbuchel H (2018) Evidence and indications for percutaneous closure of the left atrial appendage. Rev Esp Cardiol (Engl Ed) . 2018; 71:700-702.

7. Qamar SR, Jalal S, Nicolaou S, Tsang M, Gilhofer T, Saw J (2019) Comparison of cardiac computed tomography angiography and transoesophageal echocardiography for device surveillance after left atrial appendage closure. Euro Intervention. 2019; 15: 663-670.

8. Casu G, Gulizia MM, Molon G, Mazzone P, Audo A, Casolo G, Di Lorenzo E, Portoghese M, Pristipino C, Ricci RP, Themistoclakis S, Padeletti L, Tondo C, Berti S, Oreglia JA, Gerosa G, Zanobini M, Ussia GP, Musumeci G, Romeo F, Di Bartolomeo R (2017) ANMCO/AIAC/SICl-

GISE/SIC/SICCH Consensus Document: percutaneous occlusion of the left atrial appendage in nonvalvular atrial fibrillation patients: indications, patient selection, staff skills, organisation, and training. Eur Heart J 19(Suppl D): D333-D353.

9. Price MJ. Left Atrial Appendage Occlusion: Data Update. Interv Cardiol Clin. . 2018;7(2):159-168.

10. Saw J, Fahmy P, Spencer R, Prakash R, McLaughlin P, Nicolaou S, Tsang M (2016) Comparing measurements of CT angiography, TEE, and fluoroscopy of the left atrial appendage for percutaneous closure. J Cardiovasc Electrophysiol. 2016; 27: 414-422.

11. Litwinowicz R, Witowski J, Sitkowski M, Filip G, Bochenek M, Michalski M, Banaszkiewicz K, Urbanczyk-Zawadzka M, Banys R, Sobczynski R, Kapelak B, Bartus K (2018) Applications of lowcost 3D printing in left atrial appendage closure using epicardial approaches-initial clinical experience. Kardiochir Torakochirurgia Pol 15: 135-140.

12. Chow DH, Bieliauskas G, Sawaya FJ, Millan-Iturbe O, Kofoed KF, Søndergaard L, De Backer O (2017) A comparative study of different imaging modalities for successful percutaneous left atrial appendage closure. Open Heart 4: e000627.

\section{Tables}

Due to technical limitations, table 1-2 is only available as a download in the Supplemental Files section. 


\section{Figures}
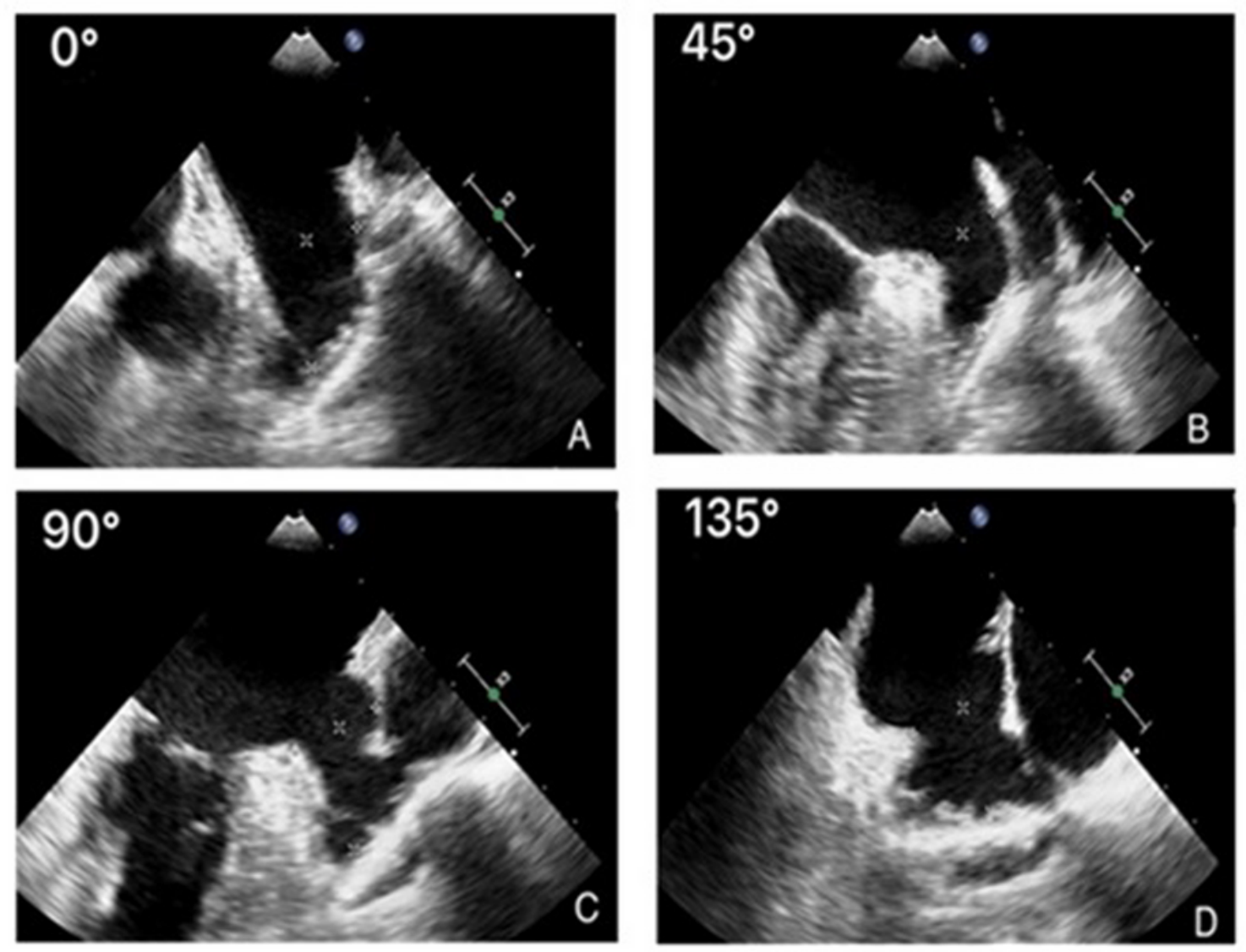

Figure 1

The orificediameter and depth of the left atrial appendage opening measured at TEE anglesof $0^{\circ}(\mathrm{A}), 45^{\circ}$ (B), $90^{\circ}(\mathrm{C})$, and $135^{\circ}(\mathrm{D})$.


Figure 2 
Preoperative CT angiography measurements of the diameter and depth of the left atrial appendage ostium. A: Image of the left atrial appendage ostium in oblique coronal view. B: Orthogonal view of A. C: Cross-sectional view of $A$.

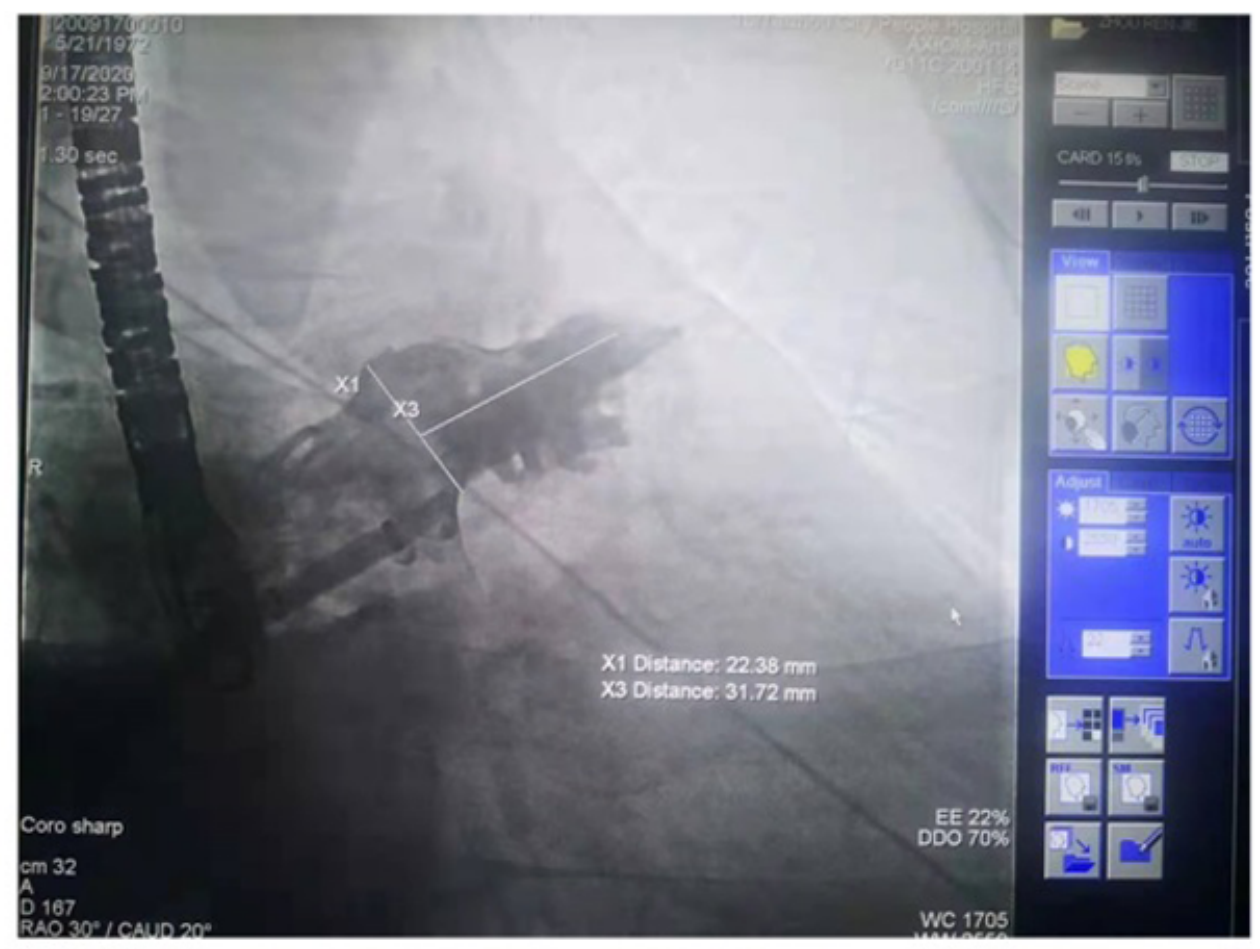

\section{Figure 3}

Intraoperative digital subtraction angiography to measure the diameter and depth of the orifice of the left atrial appendage at the angle of $\mathrm{RAO} 30^{\circ}$ andCAU20 ${ }^{\circ}$. 

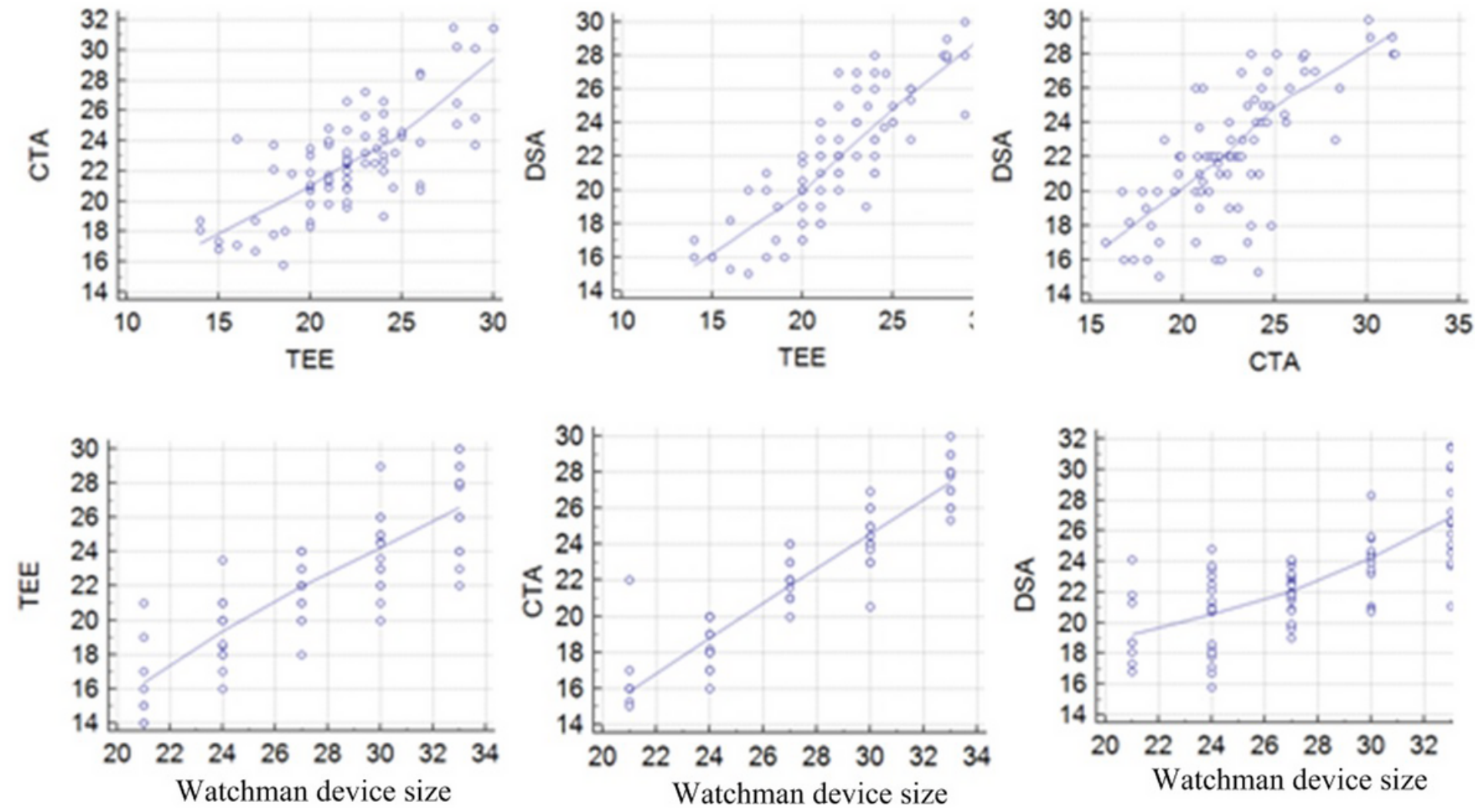

\section{Figure 4}

Correlation of LAA ostium measurements between the 3 modalities and correlation between the LAA ostium measured by 3 modalities and the Watchman device size: (A) scatter plot of TEE versus CTA, (B) scatter plot of DSA versus CTA, (C) scatter plot of TEE versus DSA, (D) the LAA ostium measured by TEE versus the Watchman device size, (E) the LAA ostium measured by CTA versus the Watchman device size, $(F)$ the LAA ostium measured by DSA versus the Watchman device size. 


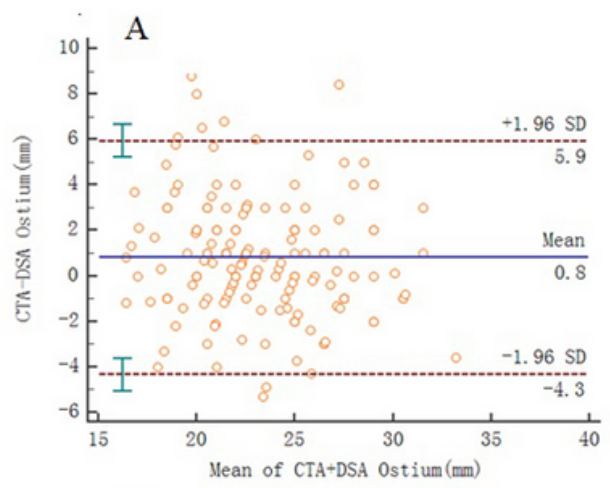

$\mathrm{D}$

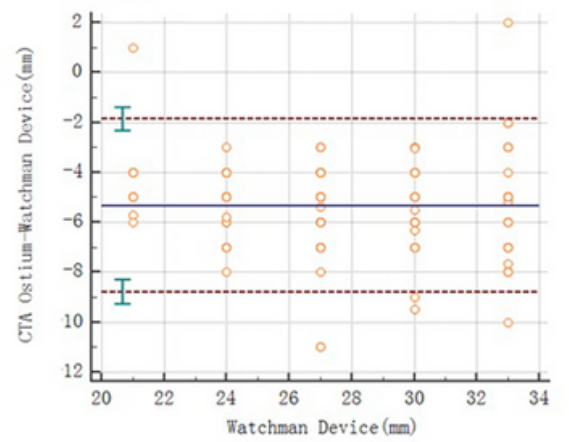

B

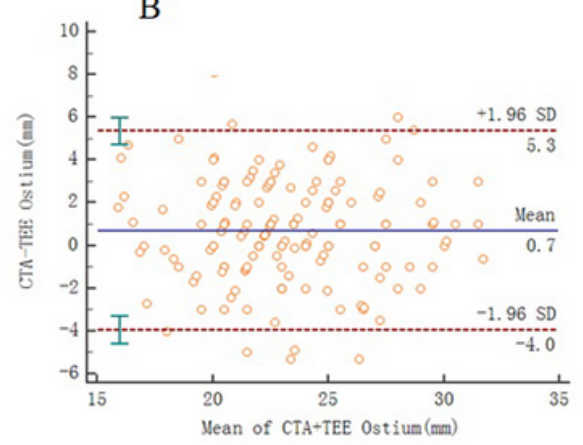

E

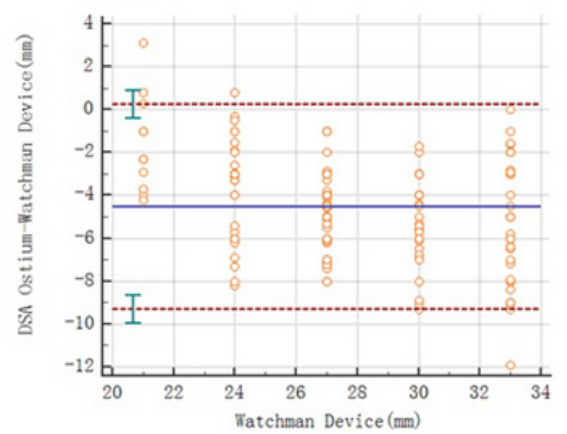

$\mathrm{C}$


Figure 5

Agreement of LAA ostium measurements between the 3 modalitie analyzed by Bland Altman diagram:(A) Bland-Altman plot of CTA versus DSA, (B) Bland-Altman plot of CTA versus TTE, (C) Bland-Altman plot of DSA versus TTE, (D) Bland-Altman plot of CTA versus Watchman device, (E) Bland-Altman plot of DSA versus Watchman device, (F) Bland-Altman plot of TEE versus Watchman device.

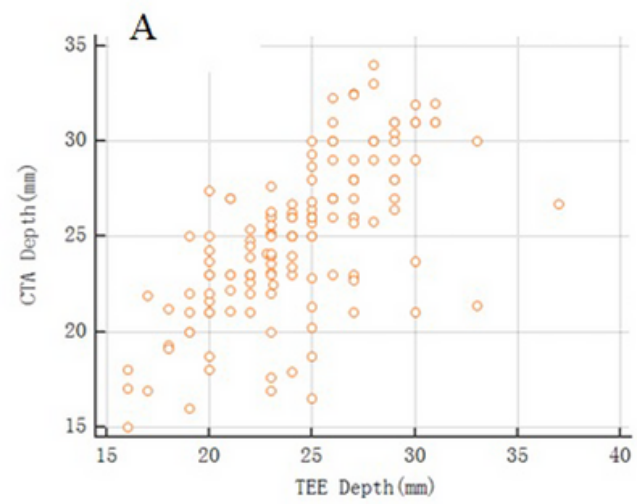

B

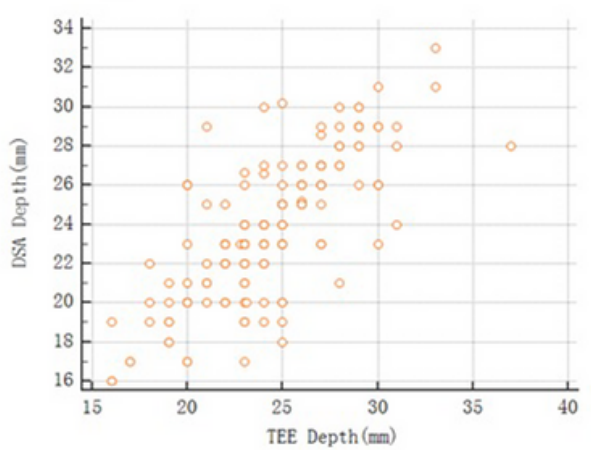

$\mathrm{C}$

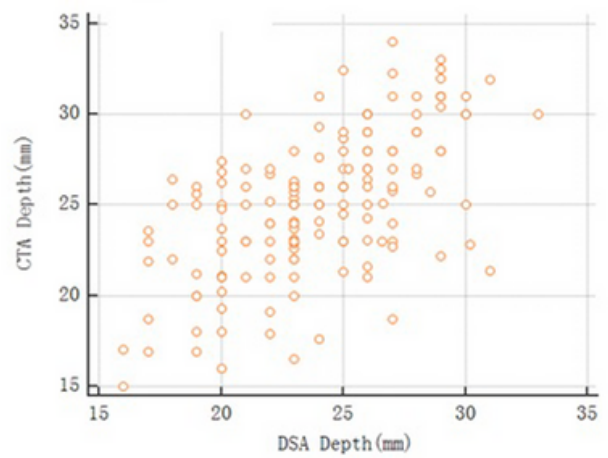

Figure 6

Correlation of LAA depth measurements between the 3 modalities: (A) scatter plot of CTA versus TEE measurements,(B) scatter plot of DSA versus TEE, (C) scatter plot of CTA versus DSA.

\section{Supplementary Files}

This is a list of supplementary files associated with this preprint. Click to download. 
- Table1.pdf

- Table2.pdf

Page 13/13 